\title{
LABA/LAMA combination in COPD: a meta-analysis on the duration of treatment
}

\author{
Luigino Calzetta ${ }^{1}$, Paola Rogliani ${ }^{1,2}$, Josuel Ora², Ermanno Puxeddu ${ }^{1}$, \\ Mario Cazzola ${ }^{1}$ and Maria Gabriella Matera ${ }^{3}$
}

Affiliations: ${ }^{1}$ Dept of Systems Medicine, University of Rome Tor Vergata, Rome, Italy. ${ }^{2}$ Division of Respiratory Medicine, University Hospital Tor Vergata, Rome, Italy. ${ }^{3}$ Dept of Experimental Medicine, Second University of Naples, Naples, Italy.

Correspondence: Luigino Calzetta, Dept of Systems Medicine, University of Rome Tor Vergata, Via Montpellier 1, 00133 Rome, Italy. E-mail luigino.calzettađuniroma2.it

@ERSpublications

We propose a simple and effective meta-analytic approach for exploring the impact of LABA/LAMA combinations in COPD http://ow.ly/8Zd9302154B

Cite this article as: Calzetta L, Rogliani P, Ora J, et al. LABA/LAMA combination in COPD: a meta-analysis on the duration of treatment. Eur Respir Rev 2017; 26: 160043 [https://doi.org/10.1183/ 16000617.0043-2016].

ABSTRACT When there are no randomised clinical trials directly comparing all relevant treatment options, an indirect treatment comparison via meta-analysis of the available clinical evidence is an acceptable alternative. However, meta-analyses may be very misleading if not adequately performed. Here, we propose and validate a simple and effective approach to meta-analysis for exploring the effectiveness of long-acting $\beta_{2}$-agonist (LABA)/long-acting muscarinic antagonist (LAMA) fixed-dose combinations in chronic obstructive pulmonary disease.

14 articles with 20329 patients (combinations $n=9292$; monocomponents $n=11037$ ) were included in this study. LABA/LAMA combinations were always more effective than the monocomponents in terms of the improvement in trough forced expiratory volume in $1 \mathrm{~s}$, transition dyspnoea index and St George's Respiratory Questionnaire scores after 3,6 and 12 months of treatment. No significant publication bias was identified. Significant discrepancies with previous network meta-analyses have been found, with overall differences ranging from $26.7 \%$ to $43.3 \%$.

Results from previous network meta-analyses were misleading because no adequate attention was given to formulating the review question, specifying eligibility criteria, correctly identifying studies, collecting appropriate information and deciding what it would be pharmacologically relevant to analyse. The real gradient of effectiveness of LABA/LAMA fixed-dose combinations remains an unmet medical need; however, it can be investigated indirectly using a high-quality meta-analytic approach.

\section{Introduction}

The wide availability of long-acting $\beta_{2}$-agonist (LABA)/long-acting muscarinic antagonist (LAMA) fixed-dose combinations (FDCs) in the absence of head-to-head comparative randomised clinical trials (RCTs) makes difficult the choice of combination for the treatment of chronic obstructive pulmonary

This article has supplementary material available from err.ersjournals.com

Received: April 272016 | Accepted after revision: June 242016

Support statement: This study was supported by institutional funds (University of Rome Tor Vergata).

Conflict of interest: Disclosures can be found alongside this article at err.ersjournals.com

Provenance: Submitted article, peer reviewed.

Copyright OERS 2017. ERR articles are open access and distributed under the terms of the Creative Commons Attribution Non-Commercial Licence 4.0. 
disease (COPD), a heterogeneous disorder characterised by an enormous variability in drug response between phenotypes $[1,2]$.

Some meta-analyses have been undertaken in an attempt to improve the gaps in our knowledge in this field [3-5]. Since no RCTs exist that have directly compared all relevant treatment options, an indirect treatment comparison via a meta-analytic approach can be an acceptable alternative to quantifying scientific uncertainty. However, only the meta-analysis published by Ова et al. [4] has investigated the impact of LABA/LAMA FDCs in COPD with specific regard to the duration of treatment. These authors performed a sophisticated meta-analysis [4], but we have identified several critical points in their method that have affected the results and produced quite inconsistent conclusions.

In particular, we strongly believe that comparing LABA/LAMA FDCs with placebo is anachronistic. In fact, there is solid evidence suggesting that the so-called "dual" bronchodilator therapy has an important role in optimising bronchodilation [6-8]. In any case, the effectiveness of dual bronchodilation should always be compared with at least one of the monocomponents included in the FDCs, and not with different LABAs or LAMAs [5]. We also believe that the regimen of administration should be consistent between the drugs of each combination, i.e. both medications should be administered once daily, for example, and not one on a once-daily and the other on a twice-daily basis [5].

A correct meta-analytic approach should have both quantitative and qualitative characteristics, in order to reveal the biases, strengths and weaknesses of analysed studies [9]. Although the Jadad score rates three out of 10 dimensions of RCT quality, it represents an easy, fast and objective tool for assessing the quality of RCTs, by revealing great overlap with the Cochrane Back Review Group [10]. In spite of this, Ова et al. [4] selected the studies to be included in their network meta-analysis according to personal opinion [4]. Moreover, they omitted to examine several important outcomes after 12 months of treatment, although RCTs in which these variables were available at week 52 were included in their analysis [4].

More surprising is that the flow diagram for the identification of the included studies was not reported in accordance with the Preferred Reporting Items for Systematic Reviews and Meta-Analyses (PRISMA) statement [11].

We have recently performed an indirect treatment comparison of the currently approved LABA/LAMA FDCs in COPD [5], in which the variables have been meta-analysed regardless of the duration of the treatment. The aim of the present meta-analysis was to explore whether the duration of treatment could in some way influence the clinical effectiveness of LABA/LAMA FDCs. Therefore, we have now adequately assessed the impact of the duration of treatment with LABA/LAMA FDCs on the trough forced expiratory volume in $1 \mathrm{~s}$ (FEV1), St George's Respiratory Questionnaire (SGRQ) and transition dyspnoea index (TDI) in COPD, by extracting each variable at the end of RCTs lasting $\geqslant 3$ months and performing a high-quality meta-analysis.

\section{Materials and methods \\ Search strategy}

This systematic review was performed in agreement with the PRISMA statement (supplementary table S1) [12].

We performed a comprehensive literature search for RCTs written in English regarding the impact of treatment with LABAs and LAMAs administered in combination in patients suffering from COPD and lasting $\geqslant 3$ months $[13,14]$.

The terms "chronic obstructive pulmonary disease" and "COPD" were searched for the disease, the terms "LABAs" and "LAMAs" for the class of drugs, the terms "aclidinium", "formoterol", "glycopyrronium", "indacaterol", "olodaterol", "tiotropium", "umeclidinium" and "vilanterol" for specific compounds and the term "combination" to identify RCTs investigating combination therapy. The search was performed on PubMed and Google Scholar in order to provide for relevant studies published up to April 15, 2016 [15]. Further searches were performed on www.clinicaltrials.gov, the EU Clinical Trials Register and the 2015 European Respiratory Society (ERS) International Congress abstracts in order to find RCTs not yet published. Citations of previous published meta-analyses and relevant reviews were checked to select further pertinent studies, if any [16-19].

\section{Study selection}

RCTs involving COPD patients that had received inhalant administration of LABA/LAMA combinations versus at least one monocomponent were included in the analysis. Two reviewers independently examined the RCTs found from literature and databases, and any difference in opinion about eligibility was resolved by consensus. 
Quality score and risk of bias assessment

The Jadad score, with a scale of 1-5 (score of 5 being the best quality), was used to assess the quality of the papers concerning the likelihood of bias related with randomisation, double blinding, withdrawals and dropouts [20]. Two reviewers independently assessed the quality of individual studies, and any difference in opinion about the quality score was resolved by consensus. RCTs with Jadad score $\geqslant 3$ were included in the meta-analysis.

The risk of publication bias was analysed by applying Egger's test in order to assess funnel plot symmetry through the following regression equation: SND $=a+b \times$ precision, where SND represents the standard normal deviate (treatment effect divided by its standard error), and precision represents the reciprocal of the standard error [21-24]. Evidence of asymmetry from Egger's test was considered to be significant for $\mathrm{p}<0.1$, and the graphical representation of $90 \%$ confidence bands have been presented [21-24].

\section{Data synthesis and analysis}

The end-point of this meta-analysis was to compare the impact of LABA/LAMA combinations with respective monocomponents, with regard to changes from baseline in trough FEV1, SGRQ and TDI score at specific time points (3,6 and 12 months).

We performed a pair-wise meta-analysis and a sensitivity analysis via a network meta-analytic approach in order to validate the obtained results. Since the data were selected from a series of studies performed by researchers operating independently, and a common effect size cannot be assumed, we used the random-effects model to perform the pair-wise meta-analysis in order to balance the study weights and to adequately estimate the confidence interval of the mean distribution of drugs effect on the investigated variables [25-28]. Values are presented as mean difference and 95\% CI. Moderate to high levels of heterogeneity were considered for $\mathrm{I}^{2}>50 \%[29]$.

The network meta-analysis was performed using a full Bayesian evidence network (chains: 4; initial values scaling: 2.5; tuning iterations: 20000 ; simulation iterations: 50000 ; and tuning interval: 10), the convergence diagnostics for consistency and inconsistency was assessed using the Brooks-Gelman-Rubin method [30, 31]. Results of the network meta-analysis are presented as mean difference and $95 \%$ credible level (Crl). Due to the complex evidence structure, the inconsistency of evidence has been assessed in addition to heterogeneity obtained from the pair-wise meta-analysis. While heterogeneity represents between-study variation in the measured relative effect of a pair of treatments, inconsistency can only occur when one of the treatments has a different effect when it is compared with the others [31]. The probability that each intervention arm was the most effective was calculated by counting the proportion of iterations of the chain in which each intervention arm had the highest mean difference, and the surface under the cumulative ranking (SUCRA), which represents the summary of these probabilities, was also calculated. The SUCRA is $100 \%$ when a treatment is certain to be the best, and $0 \%$ when a treatment is certain to be the worst [32].

The percentage of differences between the summary effects resulting from network meta-analysis and pair-wise meta-analysis was calculated in order to compare the results obtained from these different meta-analytic approaches. In particular, Gini's mean absolute difference (GMD) between each observation and any other observation has been calculated as previously described [33]. The GMD is defined in terms of absolute deviations averaging differences between pairs of observations, as defined by the following equation: $\mathrm{GMD}=\sum\left|\mathrm{X}_{\mathrm{i}}-\mathrm{X}_{\mathrm{j}}\right| / \mathrm{n}[33]$.

The optimal information size was calculated as previously reported $[34,35]$, and the statistical significance was assessed for $\mathrm{p}<0.05[36,37]$.

OpenMetaAnalyst [36] was used to perform the pair-wise meta-analysis, GeMTC [38] for network meta-analysis and GraphPad Prism (La Jolla, CA, USA) software to chart the data.

\section{Results}

\section{Study characteristics}

In accordance with our hypothesis, and by applying the Jadad score for excluding low-quality studies (cut-off $\geqslant 3$ ), we identified seven RCTs (table 1) to be excluded from the meta-analysis of Ова et al. [4], and one recent study [46] to be included. The PRISMA flow diagram and the selected RCTs are reported in supplementary figure $\mathrm{S} 1$ and table 2, respectively.

Overall, the results obtained from 20329 COPD patients (LABA/LAMA FDCs, n=9292; LABAs and LAMAs administered as monocomponents, $\mathrm{n}=11037$ ) were extracted from 14 studies that reported 20 RCTs. Five studies (nine RCTs) lasted 3 months, six studies (seven RCTs) lasted 6 months and three studies (four RCTs) lasted 12 months. 
TABLE 1 Reasons of exclusion of some of the studies included into the network meta-analysis of OBA et al. [4]

First author [ref.]

AARON [39]

DAHL [40]

MALEKI-YAZDI [41]

TASHKIN [42]

Vogelmeier [43]

MAHLER [44]

Novartis Pharmaceuticals [45]

\section{Reasons for exclusion}

nconsistency of the regimen of administration (tiotropium once daily versus salmeterol twice daily) Comparison versus placebo (indacaterol/glycopyrronium versus placebol

Comparison versus a monocomponent not included in the combination (umeclidinium/vilanterol versus tiotropium) Inconsistency of the regimen of administration

(tiotropium once daily versus formoterol twice daily) Jadad score $<3$ Jadad score $<3$

Comparison versus a monocomponent not included in the combination (indacaterol/glycopyrrolate versus tiotropium]

\section{Pair-wise meta-analysis}

LABA/LAMA FDCs significantly $(\mathrm{p}<0.001)$ improved trough FEV1 $(95 \%$ CI $)$ compared with monocomponents at 3 months (versus LABAs: $+109.02(86.36-131.68) \mathrm{mL}, \mathrm{I}^{2} 38 \%, \mathrm{p}>0.05$; versus LAMAs: $+58.05(38.70-77.40) \mathrm{mL}, \mathrm{I}^{2}$ 56\%, $\left.\mathrm{p}<0.01\right), 6$ months (versus LABAs: +72.65 (53.81-91.49) mL, $\mathrm{I}^{2} 60 \%$, $\mathrm{p}<0.05$; versus LAMAs: $\left.+40.53(15.15-65.91) \mathrm{mL}, \mathrm{I}^{2} 78 \%, \mathrm{p}<0.001\right)$ and 12 months (versus LABAs: +80.51 (66.81-94.20) mL, $\mathrm{I}^{2}$ 17\%, p>0.05; versus LAMAs: $\left.+55.65(47.26-64.05) \mathrm{mL}, \mathrm{I}^{2} 0 \%, \mathrm{p}>0.05\right)$ of treatment, although LAMAs were significantly $(\mathrm{p}<0.05)$ superior to LABAs at 3 and 12 months (figure 1 and supplementary figures S3 and S4).

Overall, the LABA/LAMA FDCs were always significantly $(\mathrm{p}<0.001)$ more effective in improving SGRQ, when compared with monocomponents (3 months: -2.04 ( $-2.59--1.49), \mathrm{I}^{2} 0 \%, \mathrm{p}>0.05$; 6 months: -1.45 $(-1.92-0.98), \mathrm{I}^{2} 8 \%, \mathrm{p}>0.05 ; 12$ months: $\left.-0.96(-1.71--0.22), \mathrm{I}^{2} 62 \%, \mathrm{p}<0.05\right)$. In addition, LABA/ LAMA FDCs significantly $(\mathrm{p}<0.001)$ improved TDI at all time points ( 3 months: $+0.67(0.45-0.89), \mathrm{I}^{2} 0 \%$, p $>0.05 ; 6$ months: $+0.41(0.30-0.51), \mathrm{I}^{2} 0 \%, \mathrm{p}>0.05 ; 12$ months: $\left.+0.39(0.21-0.56), \mathrm{I}^{2} 59 \%, \mathrm{p}<0.05\right)$, when compared with monocomponents (figure 2 and supplementary figures S5-S10).

\section{Network meta-analysis}

LABA/LAMA FDCs significantly $(\mathrm{p}<0.001)$ improved trough FEV1 $(95 \%$ CrI $)$ compared with monocomponents at 3 months (versus LABAs: +103.53 (73.40-134.74) mL; versus LAMAs: +62.82 (38.1087.09) $\mathrm{mL}$ ), 6 months (versus LABAs: +66.70 (41.45-90.87) mL; versus LAMAs: $+38.00(13.04-62.19) \mathrm{mL})$ and 12 months (versus LABAs: +81.21 (67.22-95.77) mL; versus LAMAs: +55.78 (45.90-65.91) mL).

The LABA/LAMA FDCs were significantly $(\mathrm{p}<0.001)$ more effective in improving SGRQ (95\% CrI), when compared with monocomponents, at 3 months (versus LABAs: -1.90 (-3.21--0.65); versus LAMAs: -1.99 $(-2.80--1.14)$ ), 6 months (versus LABAs: -1.57 (-2.28--0.72); versus LAMAs: $-1.33(-2.08--0.59)$ ) and 12 months versus LABAs $(-1.84(-3.63--0.20))$, but not versus LAMAs $(-0.62(-1.99-0.41))$.

In addition, LABA/LAMA FDCs significantly $(\mathrm{p}<0.001)$ improved TDI $(95 \% \mathrm{CrI})$ at 3 months (versus LABAs: +0.68 (0.20-1.17); versus LAMAs: +0.64 (0.21-1.09)), 6 months (versus LABAs: $+0.42(0.29-0.57)$; versus LAMAs: $+0.37(0.23-0.52))$ and 12 months (versus LABAs: $+0.59(0.20-1.01)$; versus LAMAs: +0.33 (0.01-0.67)), when compared with monocomponents.

Detailed results of network meta-analysis, including variance and inconsistency models, are reported in supplementary table S2.

LABA/LAMA FDCs always showed the highest probability of being the best therapy with regard of trough FEV1, SGRQ and TDI at all time points (overall 98.67\%) as confirmed by SUCRA (overall 99.28\%), whereas LAMA and LABA as monotherapy were ranked the second and third therapies (table 3).

\section{Heterogeneity, publication bias and optimal information size}

Although substantial heterogeneity due to the small-study effect was detected for some summary effects, Egger's test did not indicate any significant ( $p>0.1)$ publication bias (supplementary figure S11). In effect, in contrast with the overall test of heterogeneity, Egger's test represents a powerful tool for assessing a specific type of heterogeneity leading to publication bias. However, since any analysis of heterogeneity 


\begin{tabular}{|c|c|c|c|c|c|c|c|c|c|c|c|c|}
\hline Study; first author [ref.] & Study characteristics & $\begin{array}{c}\text { Duration } \\
\text { of study weeks }\end{array}$ & $\begin{array}{l}\text { Patients } \\
\text { analysed }\end{array}$ & Drugs & $\begin{array}{l}\text { Regimen of } \\
\text { administration }\end{array}$ & $\begin{array}{c}\text { Patient } \\
\text { characteristics }\end{array}$ & $\begin{array}{l}\text { Age } \\
\text { years }\end{array}$ & Male $\%$ & $\begin{array}{l}\text { Current } \\
\text { smokers } \\
\%\end{array}$ & $\begin{array}{l}\text { Smoking } \\
\text { history } \\
\text { pack-years }\end{array}$ & $\begin{array}{l}\text { Post-bronchodilator } \\
\text { FEV } \% \text { pred }\end{array}$ & $\begin{array}{l}\text { Jadad } \\
\text { score }\end{array}$ \\
\hline $\begin{array}{l}\text { NCT01604278; } \\
\text { VINCKEN [47] }\end{array}$ & $\begin{array}{l}\text { Multicentre, randomised, } \\
\text { double-blind, parallel-group, } \\
\text { placebo-controlled }\end{array}$ & 12 & 432 & $\begin{array}{l}\text { Glycopyrronium/ } \\
\text { indacaterol }\end{array}$ & Once daily & $\begin{array}{l}\text { Moderate to severe } \\
\text { stable COPD } \\
\text { (post-bronchodilator } \\
\mathrm{FEV}_{1} / \mathrm{FVC}<0.7 ; \mathrm{FEV} 1 \\
\geqslant 30 \% \text { and }<80 \% \text { pred) }\end{array}$ & 63.8 & 48.2 & 51.1 & 44.5 & 54.9 & 4 \\
\hline $\begin{array}{l}\text { NCT01323660, } \\
\text { NCT01328444; } \\
\text { MALTAIS [48] }\end{array}$ & $\begin{array}{l}\text { Multicentre, randomised, } \\
\text { placebo-controlled, } \\
\text { parallel-group }\end{array}$ & 12 & 768 & $\begin{array}{l}\text { Umeclidinium/ } \\
\text { vilanterol }\end{array}$ & Once daily & $\begin{array}{l}\text { Moderate to severe } \\
\text { stable COPD } \\
\text { (post-bronchodilator } \\
\mathrm{FEV}_{1} / \mathrm{FVC}<0.7 ; \mathrm{FEV} 1 \\
\geqslant 35 \% \text { and } \leqslant 70 \% \text { pred) }\end{array}$ & 62.0 & 56.4 & 62.0 & 48.1 & 51.3 & 4 \\
\hline $\begin{array}{l}\text { NCT01694771, } \\
\text { NCT01696058; } \\
\text { ZuWALLACK [49] }\end{array}$ & $\begin{array}{l}\text { Multicentre, replicate, } \\
\text { randomised, double-blind, } \\
\text { parallel-group }\end{array}$ & 12 & 2204 & $\begin{array}{l}\text { Tiotropium/ } \\
\text { olodaterol }\end{array}$ & Once daily & $\begin{array}{l}\text { Moderate to severe } \\
\text { COPD } \\
\text { (post-bronchodilator } \\
\text { FEV } 1 / \text { FVC }<0.7 ; \text { FEV } 1 \\
\geqslant 30 \% \text { and }<80 \% \text { pred) }\end{array}$ & 64.3 & 51.7 & 49.0 & 49.0 & 53.7 & 3 \\
\hline $\begin{array}{l}\text { NCT01727141, } \\
\text { NCTO1712516; } \\
\text { MAHLER [46] }\end{array}$ & $\begin{array}{l}\text { Identical, multicentre, } \\
\text { randomised, double-blind, } \\
\text { parallel-group, placebo- and } \\
\text { active-controlled }\end{array}$ & 12 & 1511 & $\begin{array}{l}\text { Glycopyrronium/ } \\
\text { indacaterol }\end{array}$ & Twice daily & $\begin{array}{l}\text { Stable COPD } \\
\text { (post-bronchodilator } \\
\mathrm{FEV}_{1} / \mathrm{FVC}<0.7 ; \mathrm{FEV}_{1} \\
\geqslant 30 \% \text { and }<80 \% \text { pred) }\end{array}$ & 63.5 & 64.8 & 52.2 & $>10$ & 54.6 & 5 \\
\hline $\begin{array}{l}\text { NCT01964352, } \\
\text { NCT02006732; } \\
\text { SINGH [50] }\end{array}$ & $\begin{array}{l}\text { Multinational, replicate, } \\
\text { randomised, double-blind, } \\
\text { placebo-controlled, parallel } \\
\text { group }\end{array}$ & 12 & 1169 & $\begin{array}{l}\text { Tiotropium/ } \\
\text { olodaterol }\end{array}$ & Once daily & $\begin{array}{l}\text { Moderate to severe } \\
\text { COPD } \\
\text { (post-bronchodilator } \\
\mathrm{FEV}_{1} / \mathrm{FVC}<0.7 ; \mathrm{FEV} 1 \\
\geqslant 30 \% \text { and }<80 \% \text { pred) }\end{array}$ & 64.8 & 61.2 & 47.7 & $>10$ & 55.1 & 3 \\
\hline $\begin{array}{l}\text { NCT01313650; } \\
\text { Donohue [51] }\end{array}$ & $\begin{array}{l}\text { Multicentre, randomised, } \\
\text { double-blind, placebo } \\
\text { controlled, parallel-group }\end{array}$ & 24 & 1252 & $\begin{array}{l}\text { Umeclidinium/ } \\
\text { vilanterol }\end{array}$ & Once daily & $\begin{array}{l}\text { COPD } \\
\text { (post-bronchodilator } \\
\mathrm{FEV}_{1} / \mathrm{FVC}<0.7 ; \mathrm{FEV}_{1} \\
\leqslant 70 \% \text { pred) }\end{array}$ & 63.3 & 70.7 & 48.2 & 46.0 & 47.6 & 4 \\
\hline $\begin{array}{l}\text { NCT01313637; } \\
\text { CELLI [52] }\end{array}$ & $\begin{array}{l}\text { Multicentre, randomised, } \\
\text { placebo-controlled, } \\
\text { parallel-group }\end{array}$ & 24 & 1214 & $\begin{array}{l}\text { Umeclidinium/ } \\
\text { vilanterol }\end{array}$ & Once daily & $\begin{array}{l}\text { COPD } \\
\text { (post-bronchodilator } \\
\mathrm{FEV}_{1} / \mathrm{FVC}<0.7 ; \mathrm{FEV} 1 \\
\leqslant 70 \% \text { pred) }\end{array}$ & 63.1 & 65.6 & 50.8 & 44.1 & 48.3 & 4 \\
\hline $\begin{array}{l}\text { NCT01316900, } \\
\text { NCT01316913; } \\
\text { DECRAMER [53] }\end{array}$ & $\begin{array}{l}\text { Multicentre, randomised, } \\
\text { blinded, parallel-group, } \\
\text { double-dummy }\end{array}$ & 24 & 1274 & $\begin{array}{l}\text { Umeclidinium / } \\
\text { vilanterol }\end{array}$ & Once daily & $\begin{array}{c}\text { COPD Icategories B or } \\
\text { D) }\end{array}$ & 63.7 & 67.4 & 44.3 & 45.4 & 47.3 & 5 \\
\hline $\begin{array}{l}\text { NCTO1437397; } \\
\text { D'URzo [54] }\end{array}$ & $\begin{array}{l}\text { Multicentre, randomised, } \\
\text { double-blind, } \\
\text { placebo-controlled }\end{array}$ & 24 & 1337 & $\begin{array}{l}\text { Aclidinium/ } \\
\text { formoterol }\end{array}$ & Twice daily & $\begin{array}{l}\text { Moderate to severe } \\
\text { stable COPD } \\
\text { (post-bronchodilator } \\
\text { FEV } 1 / F V C<0.7 ; \text { FEV } 1 \\
\geqslant 30 \% \text { and }<80 \% \text { pred) }\end{array}$ & 64.1 & 53.3 & 51.7 & 52.5 & 53.7 & 3 \\
\hline
\end{tabular}




\begin{tabular}{|c|c|c|c|c|c|c|c|c|c|c|c|c|}
\hline Study; first author [ref.] & Study characteristics & $\begin{array}{c}\text { Duration } \\
\text { of study weeks }\end{array}$ & $\begin{array}{l}\text { Patients } \\
\text { analysed }\end{array}$ & Drugs & $\begin{array}{l}\text { Regimen of } \\
\text { administration }\end{array}$ & $\begin{array}{c}\text { Patient } \\
\text { characteristics }\end{array}$ & $\begin{array}{l}\text { Age } \\
\text { years }\end{array}$ & Male \% & $\begin{array}{c}\text { Current } \\
\text { smokers } \\
\%\end{array}$ & $\begin{array}{l}\text { Smoking } \\
\text { history } \\
\text { pack-years }\end{array}$ & $\begin{array}{l}\text { Post-bronchodilator } \\
\text { FEV } 1 \% \text { pred }\end{array}$ & $\begin{array}{l}\text { Jadad } \\
\text { score }\end{array}$ \\
\hline $\begin{array}{l}\text { NCT01462942; } \\
\text { SiNGH [55] }\end{array}$ & $\begin{array}{c}\text { Multicentre, randomised, } \\
\text { double-blind, parallel-group, } \\
\text { active- and } \\
\text { placebo-controlled }\end{array}$ & 24 & 1366 & $\begin{array}{l}\text { Aclidinium/ } \\
\text { formoterol }\end{array}$ & Twice daily & $\begin{array}{l}\text { Moderate to severe } \\
\text { COPD } \\
\text { (post-bronchodilator } \\
\mathrm{FEV}_{1} / \mathrm{FVC}<0.7 ; \mathrm{FEV}_{1} \\
\geqslant 30 \% \text { and }<80 \% \text { pred) }\end{array}$ & 63.2 & 67.6 & 47.3 & $>10$ & 54.3 & 4 \\
\hline $\begin{array}{l}\text { NCT01202188; } \\
\text { BateMAN [56] }\end{array}$ & $\begin{array}{c}\text { Multicentre, randomised, } \\
\text { double-blind, parallel-group, } \\
\text { placebo- and } \\
\text { active-controlled }\end{array}$ & 26 & 1423 & $\begin{array}{l}\text { Glycopyrronium/ } \\
\text { indacaterol }\end{array}$ & Once daily & $\begin{array}{l}\text { Moderate to severe } \\
\text { stable COPD } \\
\text { (post-bronchodilator } \\
\mathrm{FEV}_{1} / \mathrm{FVC}<0.7 ; \mathrm{FEV} 1 \\
\geqslant 30 \% \text { and }<80 \% \text { pred) }\end{array}$ & 64.0 & 77.2 & 40.0 & $>10$ & 55.2 & 4 \\
\hline $\begin{array}{l}\text { NCT01431274, } \\
\text { NCT01431287; } \\
\text { BuнL [57] }\end{array}$ & $\begin{array}{l}\text { Multicentre, multinational, } \\
\text { replicate, randomised, } \\
\text { double-blind, } \\
\text { active-controlled, five-arm, } \\
\text { parallel-group }\end{array}$ & 52 & 5094 & $\begin{array}{l}\text { Tiotropium/ } \\
\text { olodaterol }\end{array}$ & Once daily & $\begin{array}{l}\text { Moderate to very severe } \\
\text { COPD } \\
\text { (post-bronchodilator } \\
\mathrm{FEV}_{1} / \mathrm{FVC}<0.7 \text {; and FEV } \\
<80 \% \text { pred) }\end{array}$ & 64.0 & 72.9 & 37.0 & $>10$ & 50.0 & 3 \\
\hline $\begin{array}{l}\text { NCT01316887; } \\
\text { Donohue [58] }\end{array}$ & $\begin{array}{l}\text { Multicentre, randomised, } \\
\text { double-blind, } \\
\text { placebo-controlled, } \\
\text { parallel-group }\end{array}$ & 52 & 177 & $\begin{array}{l}\text { Umeclidinium/ } \\
\text { vilanterol }\end{array}$ & Once daily & $\begin{array}{c}\text { COPD } \\
\text { (post-bronchodilator } \\
\mathrm{FEV}_{1} / \mathrm{FVC}<0.7 ; \mathrm{FEV}_{1} \\
\geqslant 35 \% \text { and } \leqslant 80 \% \text { pred) }\end{array}$ & 61.6 & 63.9 & NA & 41.5 & 54.6 & 4 \\
\hline $\begin{array}{l}\text { NCT01120691; } \\
\text { WEDZICHA [59] }\end{array}$ & $\begin{array}{l}\text { Multicentre, randomised, } \\
\text { double-blind, parallel-group }\end{array}$ & 64 & 1108 & $\begin{array}{l}\text { Glycopyrronium/ } \\
\text { indacaterol }\end{array}$ & Once daily & 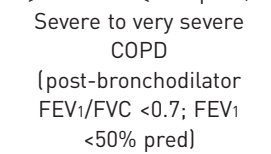 & 63.3 & 75.0 & 36.6 & $>10$ & 37.2 & 5 \\
\hline
\end{tabular}

Data are presented as $n$, unless otherwise stated. FEV1: forced expiratory volume in 1 s; COPD: chronic obstructive pulmonary disease; FVC: forced vital capacity; NA: not available. 


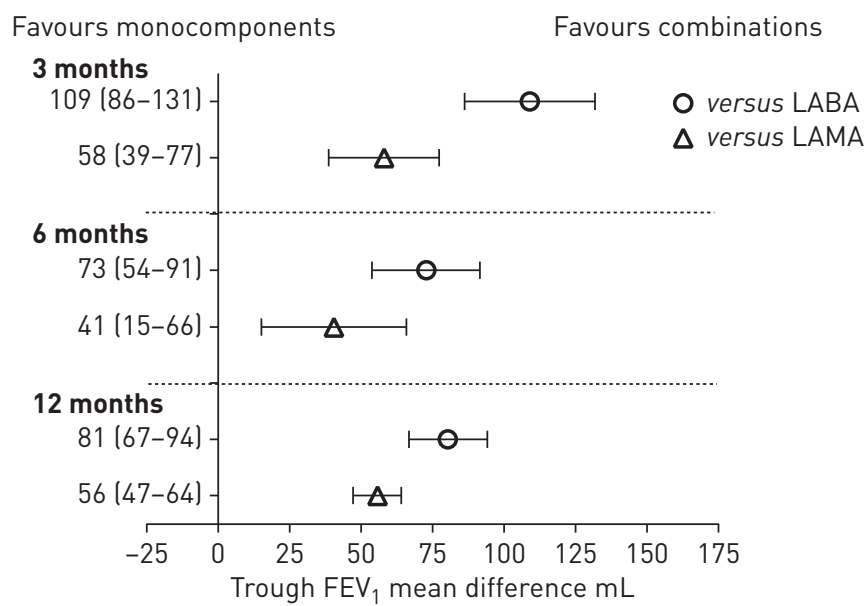

FIGURE 1 Forest plot meta-analysis of the impact of long-acting $\beta_{2}$-agonist (LABA)/long-acting muscarinic antagonist (LAMA) fixed-dose combinations on changes in trough forced expiratory volume in $1 \mathrm{~s}$ (FEV1) at 3,6 and 12 months. Data are presented as mean difference $(95 \% \mathrm{Cl})$ versus monocomponents.

depends on the number of RCTs included in the analysis, and meta-analysis with small number of studies may limit the statistical power of the test, we based evidence for bias on $\mathrm{p}<0.1$.

In addition, our meta-analysis met a reasonable optimal information size to ensure a very good (probability of observing 30\% overestimation for $\tau^{2}=0.25:<1 \%$ at true relative risk reduction $10 \%$ ) to excellent (probability of observing 20\% overestimation for $\tau^{2}=0.05:<1 \%$ at true relative risk reduction $0 \%$ ) low risk of observing an overestimated intervention effect due to random errors, and the exact numbers of optimal information size for all outcomes and at each time point are provided in supplementary table S3.

\section{Sensitivity analysis}

The sensitivity analysis indicated that the results obtained in our network meta-analysis were consistent with those of our pair-wise meta-analysis (GMD: overall 4.25\%, FEV1 5.65\%, SGRQ 4.34\% and TDI 2.76\%).

In contrast, significant discrepancy $(\mathrm{p}<0.05)$ was detected between the results of our meta-analysis and that of Ова et al. [4]. The overall GMD was 26.68\% (FEV1 19.02\%, SGRQ 19.39\% and TDI 41.62\%), and the overall discrepancy increased to $43.33 \%$ (FEV1 32.91\%, SGRQ 32.60\% and TDI $64.49 \%$ ) when the data missed by Ова et al. [4] at 12 months were included into the analysis.

\section{Discussion}

The results of our meta-analysis, which has not been affected by any publication bias, support the evidence that the dual bronchodilation with a LABA and a LAMA is more effective than monocomponents [5-8]

a) Favours

combinations

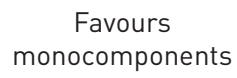

3 months

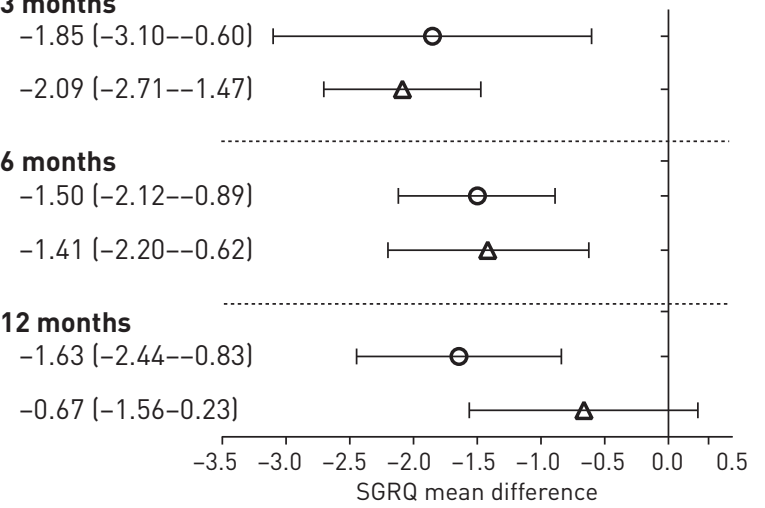

b) Favours

monocomponents

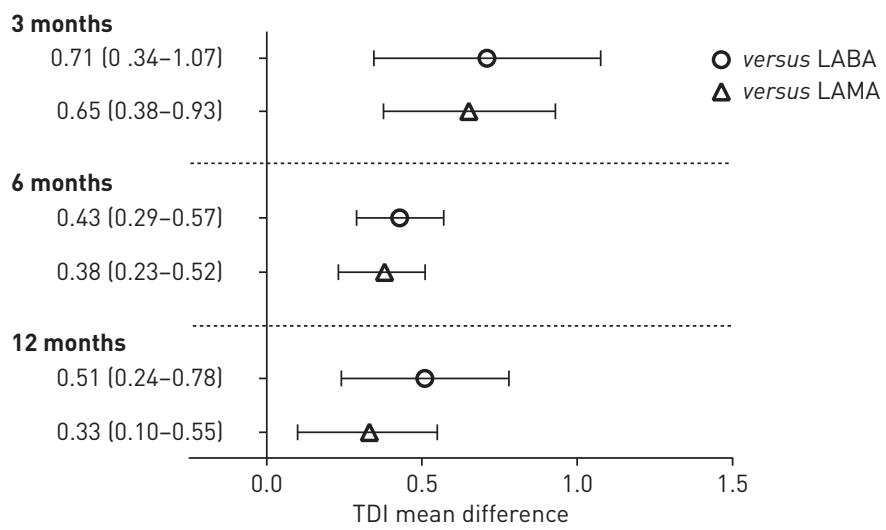

FIGURE 2 Forest plot meta-analysis of the impact of long-acting $\beta_{2}$-agonist (LABA)/long-acting muscarinic antagonist (LAMA) fixed-dose combinations on a) St George's Respiratory Questionnaire score (SGRQ) and b) transition dyspnoea index (TDI) at 3,6 and 12 months. Data are presented as mean difference and $95 \% \mathrm{Cl}$ versus monocomponents. 
TABLE 3 Probability of treatments being the best therapy and surface under the cumulative ranking curve (SUCRA) values

Trough FEV 1
3 months
LABA/LAMA
LABA
LAMA
6 months
LABA/LAMA
LABA
LAMA
12 months
LABA/LAMA
LABA
LAMA
SGRQ
3 months
LABA/LAMA
LABA
LAMA
6 months
LABA/LAMA
LABA
LAMA
12 months
LABA/LAMA
LABA
LAMA
TDI
3 months
LABA/LAMA
LABA
LAMA
LABA/LAMA
LABA
LAMA
LABA/LAMA
LAMA

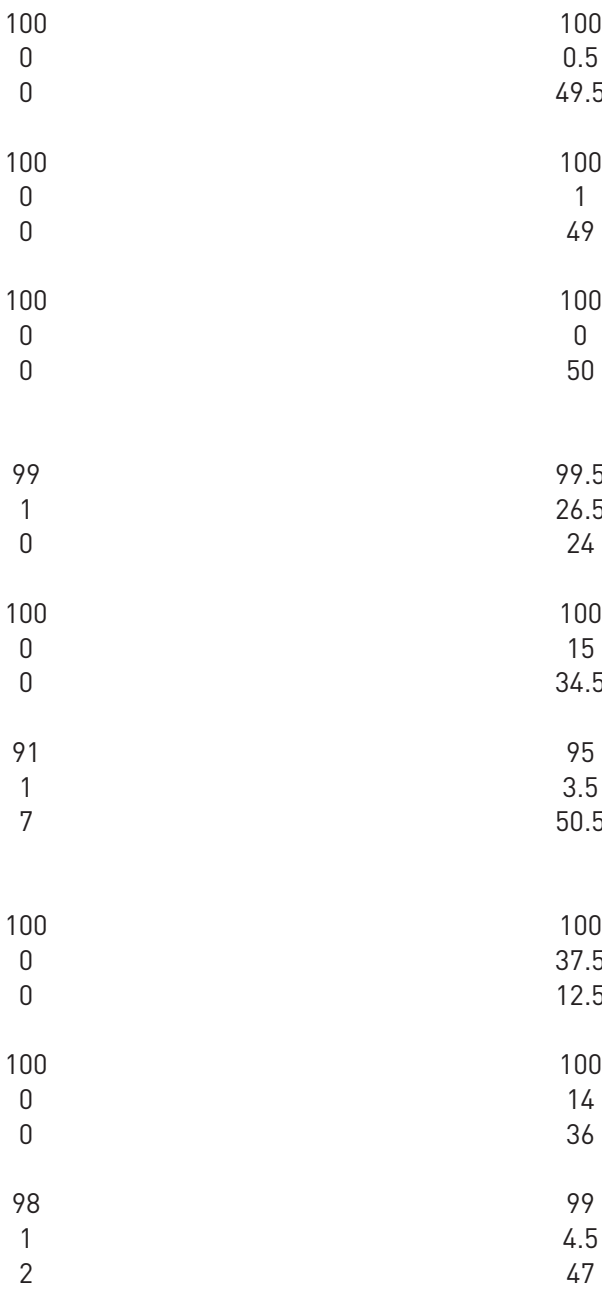

Data are presented as \%. FEV 1 : forced expiratory volume in $1 \mathrm{~s}$; LABA: long-acting $\beta_{2}$-agonist; LAMA long-acting muscarinic antagonist; SGRQ: St George's Respiratory Questionnaire; TDI: transition dyspnoea index.

after 3, 6 and 12 months of treatment. In fact, the improvement of trough FEV 1 induced by LABA/LAMA FDCs versus monotherapy was greater than the minimal clinically important difference (MCID) of $60 \mathrm{~mL}$ between active comparators (as defined by the European Medicines Agency guidelines on clinical investigation of medicinal products in the treatment of COPD), at least with regard to LABAs administered as monocomponents [60]. Although some of the analysed RCTs investigated the improvement of SGRQ scores and TDI induced by LABA/LAMA FDCs versus monotherapy, to date no published consensus exists for the MCID thresholds between active comparators for these variables. Thus, it was expected that the effect estimates resulting from our meta-analysis indicated that LABA/LAMA FDCs were unable to improve SGRQ scores and TDI more than the MCIDs (four units and one unit, respectively), when compared with monocomponents. In any case, we cannot rule out that specific LABA/LAMA FDCs may have different influences on the proportion of responder patients with regard of SGRQ and TDI, when compared with monotherapy.

Indeed, this study represents the natural step forward from our previous meta-analysis [5], in which we identified a gradient of effectiveness among the currently approved LABA/LAMA FDCs. The novel 
findings provided here support evidence that the dual bronchodilation by LABA/LABA FDCs is more effective than that elicited by monocomponents, especially with regard to LABAs for changes from baseline in trough FEV1. Furthermore, the overall superiority of LABA/LAMA FDCs was greater after 3 months of treatment, while it diminished after 6 and 12 months of treatment. This trend suggests that continued improvements in FEV1 elicited by LABA/LAMA combinations can be expected over the first 3 months of treatment; after that, the greater benefits of dual bronchodilation remains stable. Thus, it seems that for long-acting bronchodilator agents the time taken to reach the clinical bronchorelaxant steady state is considerably longer than the time taken to achieve the pharmacodynamic steady state [61, $62]$, meaning that the LABA/LAMA interaction is fundamental, not only after acute administration $[7,8]$, but also over time in the course of chronic treatment.

We must highlight that our results are considerably different from those obtained by Ова et al. [4], with an overall discrepancy of $\approx 26 \%$ with regard to FEV1, SGRQ and TDI variables. This incongruity reached $\approx 43 \%$ if the data missed by Ова et al. [4] at 12 months were included in the analysis. Although we cannot understand why data at 12 months were omitted, we suppose that the relevant inconsistencies between our results and those published by Ова et al. [4] may be related to an inadequate selection of RCTs, an inappropriate extraction of raw data from published papers and an inaccurate consultation of repository databases such as clinicaltrials.gov. In addition, the authors [4] used a sophisticated network meta-analytic approach that may have led to frequent inconsistent and biased results [63].

However, in order to dispel any doubt, we have performed a sensitivity test by performing a network meta-analysis on the 14 studies included in the quantitative synthesis. Intriguingly, we have detected only $\approx 4 \%$ difference when compared with our pair-wise meta-analysis. This finding suggests that the distance between our results and those of Ова et al. [4] cannot be strictly attributable to the difference between the pair-wise and network meta-analytic approach.

We fully agree with DeEKs et al. [64] that the production of a diamond at the bottom of a plot is an exciting moment for any researchers. Nevertheless, results of meta-analyses can be very misleading if adequate attention has not been given to formulating the review question, specifying eligibility criteria, identifying, selecting and critically appraising studies, collecting appropriate data and deciding what would be meaningful to analyse [64].

In the light of these evidences, and considering the inaccurate network meta-analysis performed by OвА et al. [4], our pair-wise meta-analysis provides an alternative view of the examination of dual bronchodilation with regard to the duration of treatment, based on the evaluation of the pharmacological characteristics of bronchodilator agents and performed through simpler meta-analytic methods.

We strongly believe that the effectiveness of dual bronchodilation should always be compared with at least one of the monocomponents included in the FDCs, and not with different LABAs or LAMAs. In fact, it should be obvious that comparing LABA/LAMA combinations with monocomponents characterised by different pharmacokinetics and pharmacodynamics represents a strict pharmacological matter [65, 66], and not a merely statistical problem that may be solved by performing a subgroup analysis. Furthermore, we believe that the regimen of administration should be consistent between the drugs of each combination, i.e. both medications administered once daily or twice daily, and not at different time intervals.

Finally, several LABA/LAMA FDCs have been approved for COPD, but no head-to-head comparative RCTs have yet been conducted. We strongly believe that the real gradient of effectiveness of the approved LABA/ LAMA FDCs remains an unmet medical need, which can be indirectly investigated using specific and well-performed meta-analytic approaches, considering that a homogenous body of literature already exists.

\section{Acknowledgement}

We would like to thank Robert Manna (Naples, Italy) for editing this manuscript.

\section{References}

1 Cazzola M, Calzetta L, Rogliani P, et al. The discovery of roflumilast for the treatment of chronic obstructive pulmonary disease. Expert Opin Drug Discov 2016; 11: 733-744.

2 Rogliani P, Calzetta L, Cazzola M, et al. Drug safety evaluation of roflumilast for the treatment of COPD: a meta-analysis. Expert Opin Drug Saf 2016; 15: 1133-1146.

3 Schlueter M, Gonzalez-Rojas N, Baldwin M, et al. Comparative efficacy of fixed-dose combinations of long-acting muscarinic antagonists and long-acting $\beta 2$-agonists: a systematic review and network meta-analysis. Ther $A d v$ Respir Dis 2016; 10: 89-104.

4 Oba Y, Sarva ST, Dias S. Efficacy and safety of long-acting $\beta$-agonist/long-acting muscarinic antagonist combinations in COPD: a network meta-analysis. Thorax 2016; 71: 15-25.

5 Calzetta L, Rogliani P, Matera MG, et al. A systematic review with meta-analysis of dual bronchodilation with LAMA/LABA for the treatment of stable COPD. Chest 2016; 149: 1181-1196. 
6 Calzetta L, Matera MG, Cazzola M. Pharmacological interaction between LABAs and LAMAs in the airways: optimizing synergy. Eur J Pharmacol 2015; 761: 168-173.

7 Cazzola M, Calzetta L, Ora J, et al. Searching for the synergistic effect between aclidinium and formoterol: from bench to bedside. Respir Med 2015; 109: 1305-1311.

8 Cazzola M, Calzetta L, Segreti A, et al. Translational study searching for synergy between glycopyrronium and indacaterol. COPD 2015; 12: 175-181.

9 Russo MW. How to review a meta-analysis. Gastroenterol Hepatol 2007; 3: 637-642.

10 Berger VW, Alperson SY. A general framework for the evaluation of clinical trial quality. Rev Recent Clin Trials 2009; 4: 79-88.

11 Moher D, Liberati A, Tetzlaff J, et al. Preferred reporting items for systematic reviews and meta-analyses: the PRISMA statement. PLoS Med 2009; 6: e1000097.

12 Moher D, Liberati A, Tetzlaff J, et al. Preferred reporting items for systematic reviews and meta-analyses: the PRISMA Statement. Open Med 2009; 3: e123-e130.

13 Global Initiative for Chronic Obstructive Lung Disease (GOLD). Global Strategy for the Diagnosis, Management and Prevention of COPD. www.goldcopd.org/ Date last accessed: November 30, 2015. Date last updated: 2015.

14 Celli BR, MacNee W. Standards for the diagnosis and treatment of patients with COPD: a summary of the ATS/ ERS position paper. Eur Respir J 2004; 23: 932-946.

15 Shariff SZ, Bejaimal SA, Sontrop JM, et al. Retrieving clinical evidence: a comparison of PubMed and Google Scholar for quick clinical searches. J Med Internet Res 2013; 15: e164.

16 Kew KM, Dias S, Cates CJ. Long-acting inhaled therapy (beta-agonists, anticholinergics and steroids) for COPD: a network meta-analysis. Cochrane Database Syst Rev 2014; 3: CD010844.

17 Horita N, Kaneko T. Role of combined indacaterol and glycopyrronium bromide (QVA149) for the treatment of COPD in Japan. Int J Chron Obstruct Pulmon Dis 2015; 10: 813-822.

18 Malerba M, Morjaria JB, Radaeli A. Differential pharmacology and clinical utility of emerging combination treatments in the management of COPD - role of umeclidinium/vilanterol. Int J Chron Obstruct Pulmon Dis 2014; 9: 687-695.

19 Roskell NS, Anzueto A, Hamilton A, et al. Once-daily long-acting beta-agonists for chronic obstructive pulmonary disease: an indirect comparison of olodaterol and indacaterol. Int J Chron Obstruct Pulmon Dis 2014; 9: 813-824.

20 Jadad AR, Moore RA, Carroll D, et al. Assessing the quality of reports of randomized clinical trials: is blinding necessary? Control Clin Trials 1996; 17: 1-12.

21 Egger M, Davey Smith G, Schneider M, et al. Bias in meta-analysis detected by a simple, graphical test. BMJ 1997; 315: 629-634.

22 Sterne JA, Egger M. Funnel plots for detecting bias in meta-analysis: guidelines on choice of axis. J Clin Epidemiol 2001; 54: 1046-1055.

23 Sterne JA, Gavaghan D, Egger M. Publication and related bias in meta-analysis: power of statistical tests and prevalence in the literature. J Clin Epidemiol 2000; 53: 1119-1129.

24 Sterne JAC, Harbord RM. Funnel plots in meta-analysis. Stata J 2004; 4: 127-141.

25 Borenstein M. Introduction to Meta-analysis. Chichester, John Wiley \& Sons, 2009.

26 Cooper HM, Hedges LV, Valentine JC. The Handbook of Research Synthesis and Meta-analysis. 2nd Edn. New York, Russell Sage Foundation, 2009.

27 Turner JR, Durham TA. Meta-methodology: conducting and reporting meta-analyses. J Clin Hypertens 2014; 16: 91-93.

28 Cazzola M, Calzetta L, Page $\mathrm{C}$, et al. Influence of $\mathrm{N}$-acetylcysteine on chronic bronchitis or COPD exacerbations: a meta-analysis. Eur Respir Rev 2015; 24: 451-461.

29 Higgins JP, Thompson SG, Deeks JJ, et al. Measuring inconsistency in meta-analyses. BMJ 2003; 327: 557-560.

30 Brooks SP, Gelman A. General methods for monitoring convergence of iterative simulations. J Comput Graph Stat 1998; 7: 434-455.

31 Lu G, Ades AE. Assessing evidence inconsistency in mixed treatment comparisons. J Am Stat Assoc 2006; 101: 447-459.

32 Salanti G, Ades AE, Ioannidis JP. Graphical methods and numerical summaries for presenting results from multiple-treatment meta-analysis: an overview and tutorial. J Clin Epidemiol 2011; 64: 163-171.

33 Cahan S, Gamliel E. First among others? Cohen's "d" vs. alternative standardized mean group difference measures. Pract Assess Res Eval 2011; 16: 1-6.

34 Thorlund $\mathrm{K}$, Imberger G, Walsh $\mathrm{M}$, et al. The number of patients and events required to limit the risk of overestimation of intervention effects in meta-analysis - a simulation study. PLoS One 2011; 6: e25491.

35 Guyatt GH, Oxman AD, Kunz R, et al. GRADE guidelines 6. Rating the quality of evidence - imprecision. J Clin Epidemiol 2011; 64: 1283-1293.

36 Wallace BC, Dahabreh IJ, Trikalinos TA, et al. Closing the gap between methodologists and end-users: R as a computational back-end. J Stat Softw 2012; 49: 1-15.

37 Viechtbauer W. Conducting meta-analyses in R with the metafor package. J Stat Softw 2010; 36: 1-48.

38 van Valkenhoef G, Lu G, de Brock B, et al. Automating network meta-analysis. Res Synth Method 2012; 3: 285-299.

39 Aaron SD, Vandemheen KL, Fergusson D, et al. Tiotropium in combination with placebo, salmeterol, or fluticasone-salmeterol for treatment of chronic obstructive pulmonary disease: a randomized trial. Ann Intern Med 2007; 146: 545-555.

40 Dahl R, Chapman KR, Rudolf M, et al. Safety and efficacy of dual bronchodilation with QVA149 in COPD patients: the ENLIGHTEN study. Respir Med 2013; 107: 1558-1567.

41 Maleki-Yazdi MR, Kaelin T, Richard N, et al. Efficacy and safety of umeclidinium/vilanterol 62.5/25 mcg and tiotropium $18 \mathrm{mcg}$ in chronic obstructive pulmonary disease: results of a 24-week, randomized, controlled trial. Respir Med 2014; 108: 1752-1760.

42 Tashkin DP, Pearle J, Iezzoni D, et al. Formoterol and tiotropium compared with tiotropium alone for treatment of COPD. COPD 2009; 6: 17-25.

43 Vogelmeier C, Kardos P, Harari S, et al. Formoterol mono- and combination therapy with tiotropium in patients with COPD: a 6-month study. Respir Med 2008; 102: 1511-1520. 
44 Mahler DA, D'Urzo A, Bateman ED, et al. Concurrent use of indacaterol plus tiotropium in patients with COPD provides superior bronchodilation compared with tiotropium alone: a randomised, double-blind comparison. Thorax 2012; 67: 781-788.

45 Novartis Pharmaceuticals. Long-term Safety and Tolerability of QVA149 Versus Tiotropium in Japanese Patients With Chronic Obstructive Pulmonary Disease. https:/clinicaltrials.gov/ct2/show/NCT01285492 Date last accessed: November 21, 2015. Date last updated: December 3, 2013.

46 Mahler DA, Kerwin E, Ayers T, et al. FLIGHT1 and FLIGHT 2: efficacy and safety of QVA149 (indacaterol/ glycopyrrolate) versus its monocomponents and placebo in patients with chronic obstructive pulmonary disease. Am J Respir Crit Care Med 2015; 192: 1068-1079.

47 Vincken W, Aumann J, Chen H, et al. Efficacy and safety of coadministration of once-daily indacaterol and glycopyrronium versus indacaterol alone in COPD patients: the GLOW6 study. Int J Chron Obstruct Pulmon Dis 2014; 9: 215-228.

48 Maltais F, Singh S, Donald AC, et al. Effects of a combination of umeclidinium/vilanterol on exercise endurance in patients with chronic obstructive pulmonary disease: two randomized, double-blind clinical trials. Ther Adv Respir Dis 2014; 8: 169-181.

49 ZuWallack R, Allen L, Hernandez G, et al. Efficacy and safety of combining olodaterol Respimat ${ }^{\circledR}$ and tiotropium HandiHaler $^{\star}$ in patients with COPD: results of two randomized, double-blind, active-controlled studies. Int $J$ Chron Obstruct Pulmon Dis 2014; 9: 1133-1144.

50 Singh D, Ferguson GT, Bolitschek J, et al. Tiotropium + olodaterol shows clinically meaningful improvements in quality of life. Respir Med 2015; 109: 1312-1319.

51 Donohue JF, Maleki-Yazdi MR, Kilbride S, et al. Efficacy and safety of once-daily umeclidinium/vilanterol 62.5/25 mcg in COPD. Respir Med 2013; 107: 1538-1546.

52 Celli B, Crater G, Kilbride S, et al. Once-daily umeclidinium/vilanterol 125/25 mcg in COPD: a randomized, controlled study. Chest 2014; 145: 981-991.

53 Decramer M, Anzueto A, Kerwin E, et al. Efficacy and safety of umeclidinium plus vilanterol versus tiotropium, vilanterol, or umeclidinium monotherapies over 24 weeks in patients with chronic obstructive pulmonary disease: results from two multicentre, blinded, randomised controlled trials. Lancet Respir Med 2014; 2: 472-486.

54 D'Urzo AD, Rennard SI, Kerwin EM, et al. Efficacy and safety of fixed-dose combinations of aclidinium bromide/ formoterol fumarate: the 24-week, randomized, placebo-controlled AUGMENT COPD study. Respir Res 2014 ; 15 : 123.

55 Singh D, Jones PW, Bateman ED, et al. Efficacy and safety of aclidinium bromide/formoterol fumarate fixed-dose combinations compared with individual components and placebo in patients with COPD (ACLIFORM-COPD): a multicentre, randomised study. BMC Pulm Med 2014; 14: 178.

56 Bateman ED, Ferguson GT, Barnes N, et al. Dual bronchodilation with QVA149 versus single bronchodilator therapy: the SHINE study. Eur Respir J 2013; 42: 1484-1494.

57 Buhl R, Maltais F, Abrahams R, et al. Tiotropium and olodaterol fixed-dose combination versus mono-components in COPD (GOLD 2-4). Eur Respir J 2015; 45: 969-979.

58 Donohue JF, Niewoehner D, Brooks J, et al. Safety and tolerability of once-daily umeclidinium/vilanterol 125/25 mcg and umeclidinium $125 \mathrm{mcg}$ in patients with chronic obstructive pulmonary disease: results from a 52-week, randomized, double-blind, placebo-controlled study. Respir Res 2014; 15: 78.

59 Wedzicha JA, Decramer M, Ficker JH, et al. Analysis of chronic obstructive pulmonary disease exacerbations with the dual bronchodilator QVA149 compared with glycopyrronium and tiotropium (SPARK): a randomised, double-blind, parallel-group study. Lancet Respir Med 2013; 1: 199-209.

60 Bateman E. Lack of clinically relevant differences between combination therapy and monotherapy in COPD. Eur Respir J 2014; 43: 1204-1205.

61 van Noord JA, Aumann JL, Janssens E, et al. Comparison of tiotropium once daily, formoterol twice daily and both combined once daily in patients with COPD. Eur Respir J 2005; 26: 214-222.

62 Kempsford R, Norris V, Siederer S. Vilanterol trifenatate, a novel inhaled long-acting $\beta_{2}$ adrenoceptor agonist, is well tolerated in healthy subjects and demonstrates prolonged bronchodilation in subjects with asthma and COPD. Pulm Pharmacol Ther 2013; 26: 256-264.

63 Salanti G. Indirect and mixed-treatment comparison, network, or multiple-treatments meta-analysis: many names, many benefits, many concerns for the next generation evidence synthesis tool. Research Synth Methods 2012; 3: 80-97.

64 Deeks JJ, Higgins J, Altman DG. Analysing data and undertaking meta-analyses. In: Higgins JPT, Green S, eds. Cochrane Handbook for Systematic Reviews of Interventions: Cochrane Book Series. The Cochrane Collaboration, 2008; pp. 243-296.

65 Salmon M, Luttmann MA, Foley JJ, et al. Pharmacological characterization of GSK573719 (umeclidinium): a novel, long-acting, inhaled antagonist of the muscarinic cholinergic receptors for treatment of pulmonary diseases. J Pharmacol Exp Ther 2013; 345: 260-270.

66 Cazzola M, Page CP, Rogliani P, et al. $\beta_{2}$-agonist therapy in lung disease. Am J Respir Crit Care Med 2013; 187: 690-696. 